\title{
Remote effect of kidney ischemia-reperfusion injury on pancreas: role of oxidative stress and mitochondrial apoptosis
}

\author{
Noha M. Abogresha ${ }^{1}$, Sahar Mansour Greish ${ }^{1}$, Eman Z. Abdelaziz², Waleed F. Khalil ${ }^{3}$
}

\begin{abstract}
${ }^{1}$ Physiology Department, Faculty of Medicine, Suez Canal University, Ismaileya, Egypt ${ }^{2}$ Pharmacology Department, Faculty of Medicine, Suez Canal University, Ismaileya, Egypt ${ }^{3}$ Pharmacology Department, Faculty of Veterinary Medicine, Suez Canal University, Ismaileya, Egypt
\end{abstract}

Submitted: 11 August 2014

Accepted: 17 December 2014

Arch Med Sci 2016; 12, 2: 252-262

DOI: 10.5114 /aoms.2015.48130

Copyright $\odot 2016$ Termedia \& Banach

\section{Abstract}

Introduction: Recent studies have demonstrated remote effects of renal ischemia/reperfusion (IR) injury on some organs such as brain, liver, and lungs. Oxidative stress is reported to be the cornerstone in such ischemic conditions. Associated apoptosis is also reported in remote lung, liver and myocardial injury after acute kidney injury. So, we postulated that renal IR may affect the pancreas by its remote effect. Oxidative stress and mitochondrial mediated apoptosis may play a crucial role in this injury. We investigated the effects of kidney IR on pancreatic exocrine and endocrine functions, antioxidant enzyme activity, and apoptosis.

Material and methods: The protective effect of vitamin $C$ was also investigated. The animals were submitted to non-traumatic bilateral renal IR, sham operation or treatment with vitamin C after IR. Rats were sacrificed on the $1^{\text {st }}, 3^{\text {rd }}$, and $7^{\text {th }}$ days of the experiment to evaluate the parameters of oxidative stress (catalase, lipid peroxidase, reduced glutathione and superoxide dismutase), pancreatic endocrine and exocrine function (amylase, insulin and fasting blood glucose), renal functions (serum creatinine and blood urea nitrogen), cellular injury and apoptotic markers (Bcl-2, Bax and caspase-3). Results: Kidney I/R significantly increased the renal and pancreatic functions at 1,3 and 7 days, while fasting insulin was significantly increased at day 3 after ischemia. Moreover, I/R significantly increased the studied oxidative stress markers and decreased the antioxidant capacity in pancreatic tissues. In addition, renal I/R induced numerous histopatological lesions in pancreatic tissues and increased the apoptosis-related genes. Treating the rats with vitamin $C(100 \mathrm{mg} / \mathrm{kg})$ significantly restored the renal and pancreatic functions, improved the pancreatic antioxidant capacity and protected the pancreatic tissues from apoptotic necrosis.

Conclusions: The results suggested that bilateral renal ischemia for $45 \mathrm{~min}$ caused significant impairment of pancreatic function and structure as indicators of acute pancreatitis. While IR enhances oxidative stress and apoptosis, vitamin C appears to play a cytoprotective role.

Key words: kidney ischemia-reperfusion, pancreatic apoptosis, oxidative stress.

\section{Introduction}

Kidney ischemia-reperfusion (IR) injury occurs in various clinical settings including shock, sepsis, organ transplantation, vascular surgery and

\author{
Corresponding author: \\ Sahar Mansour Greish \\ Physiology Department \\ Faculty of Medicine \\ Suez Canal University, Egypt \\ 41522 Ismaileya, Egypt \\ Phone: 00201065080508 \\ E-mail: saharmg@yahoo.com
}


many other conditions [1]. Distant organ effects of acute kidney injury (AKI) are evident. Progression to multiple organ failure following AKI is the leading cause of morbidity and mortality; it is the end point of remote effects of IR injury [2]. Several studies have demonstrated that injured kidneys affect different remote organs such as lungs, liver, heart, gut, brain, and the hematologic system. However, remote effects of renal IR injury on the pancreas remain unclear [3]. Pancreatic injury as a remote organ has been reported following liver $I R$ in liver transplantation and in major liver resections [2, 3]. There is an association between IR liver injury and pancreatic injury [3]. Pancreatic injuries are difficult to diagnose and are associated with high morbidity and mortality. Although it is clear that the pathogenesis of remote organ injury is multifactorial, the formation of reactive oxygen species (ROS) is the frequently blamed mechanism [2]. Ischemia-reperfusion injury results in ROS that augment local tissue damage and affect organs remote from the site of IR [4]. Reactive oxygen species are highly unstable oxygen molecules capable of causing cellular damage primarily through the peroxidation of lipids in the membranes of the cell and the mitochondria as well as DNA degradation [2]. The role of oxidative stress in pancreatic injury has been studied in several animal models. Reactive oxygen species have been implicated as important agents in IR associated with pancreatic transplantation procedures. Moreover, it is reported that the release of ROS causes apoptosis of cells as a late phase response following IR [4]. Apoptosis has been reported in remote lung, liver and myocardial injury after AKI $[2,5]$. Reactive oxygen species are considered an important cause of death of pancreatic cells [3]. It is well documented that overproduction of oxygen free radicals in the pancreas overwhelms its endogenous antioxidants and scavenging capacity, leading to cell injury through peroxidation of the lipid component of mitochondrial and cellular membrane [4]. The endogenous antioxidants superoxide dismutase (SOD) and catalase (CAT) as well as glutathione (GSH), which are responsible for defense against ROS, have an important role in reducing IR injury [4]. Hence, antioxidants have been commonly used in ischemia and reperfusion injury and have been shown to attenuate ischemic and oxidative injuries to many tissues [2]. As the cells receive negative signals in the form of increased ROS levels, an imbalance ensues between the positive signals needed for cell survival and the increased cellular level of oxidants, so apoptosis occurs [5]. Furthermore, it is now well established that mitochondria play a key role in regulating apoptosis [6]. Oxidative stress triggers caspase-3 activation leading to cell apoptosis. In addition, the balance of anti- and proapoptotic proteins (Bcl-2: Bax) in the mitochondria responds dramatically to ROS [7]. Vitamin C plays an important role in a number of metabolic functions; it has an antioxidant property that protects the body from free radical damage. It is used as a therapeutic agent in many diseases and disorders [8].

We postulated that there is a possible remote effect of renal IR injury on the pancreas. Oxidative stress and mitochondrial mediated apoptosis may play role in this injury, and the use of vitamin C can play a cytoprotective role.

\section{Material and methods}

\section{Animals}

Fifty four male Sprague-Dawley rats (200-250 g) were used. Rats were purchased from the National Centre of Research (Cairo, Egypt), housed in clean cages under hygienic conditions and allowed to acclimatize for seven days before starting the experiment. Rats were kept on a standard chow and water ad libitum with reversed dark-light cycle. All experimental protocols were approved by the Institutional Animal Care and Use Committee at Suez Canal University. All efforts were made to minimize animal suffering and to reduce the number of animals used. The "Principles of laboratory animal care" were followed, as well as specific national laws where applicable.

\section{Experimental protocol}

Rats were randomly allocated into three groups, 18 rats each:

- Group I (Sham operation group): rats underwent identical surgical procedures as an IR group without bilateral renal clamping.

- Group II (IR) group: anesthesia was induced with ketamine $50 \mathrm{mg} / \mathrm{kg}$ intraperitoneally. The animals were placed on a heating mat, to keep the body temperature at $36 \pm 1^{\circ} \mathrm{C}$. A midline laparotomy was performed, both kidneys were located, and the renal pedicles, containing the artery, vein, and nerve supplying each kidney, were carefully isolated. Rats were allowed to stabilize for 45 min before they were subjected to bilateral renal pedicles [3]. Once reperfusion commenced the artery clips were removed. The occlusion was verified visually by a change in the color of the kidneys to a paler shade and reperfusion by a blush.

- Group III (treated group): rats underwent identical surgical procedures as IR group, and then treated with vitamin C $(100 \mathrm{mg} / \mathrm{kg})$ orally dissolved in the drinking water.

Animals were anesthetized again and sacrificed after 1 day, 3 days and 7 days after ischemia (6 animals in each experimental group at each time 
of observation). Animals were fasting overnight, fasting blood glucose level was determined before sacrifice. Blood was collected from the heart.

After the blood withdrawal, the pancreas was fully dissected out from its attachment to the stomach, duodenum and spleen. Fat and peripancreatic tissue were trimmed away. The entire pancreas was rapidly excised and sectioned into two pieces along the longitudinal axis for histological and immune-histochemical analysis.

\section{Biochemical analysis}

Renal function (serum creatinine and blood urea nitrogen (BUN)) and pancreatic function (serum amylase, lipase, fasting insulin and fasting blood glucose) were measured at day 1, 3 and 7 after ischemia. All of these measurements were done with commercially available kits (Fortress diagnostics, UK).

\section{Measurement of oxidative stress markers in pancreatic tissues}

Oxidative stress markers catalase, lipid peroxidase, reduced glutathione and superoxide dismutase (SOD) were measured in pancreatic tissues. Tissue samples were homogenized in four volumes of ice-cold Tris- $\mathrm{HCl}$ buffer $(50 \mathrm{mM}, \mathrm{pH}$ 7.4) on Homogenizer (Ultra Turrax IKA T18 Basic, USA) for $2 \mathrm{~min}$ at $5,000 \mathrm{~g}$ at $4^{\circ} \mathrm{C}$. Then the level of lipid peroxidase was measured. The homogenate was then centrifuged at $5,000 \mathrm{~g}$ at $4^{\circ} \mathrm{C}$ for $60 \mathrm{~min}$. The supernatant was collected for each assay of CAT activities. An equal volume of an ethanol/chloroform mixture ( $5: 3, \mathrm{v} / \mathrm{v})$ was added to the homogenate and the supernatant solution was extracted. This was clarified by centrifugation at $5,000 \mathrm{~g}$ at $4^{\circ} \mathrm{C}$ for $30 \mathrm{~min}$. The clear upper layer was collected and assayed for superoxide dismutase (SOD).

Lipid peroxidase level was measured by using the method of Draper and Hadley [9]. The principle of the method is the spectrophotometric measurement of the color produced during the reaction with thiobarbituric acid (TBA) with lipid peroxidase. For this purpose, $2.5 \mathrm{ml}$ of $100 \mathrm{~g} / \mathrm{l}$ trichloroacetic acid solution was added to $0.5 \mathrm{ml}$ of serum in a centrifuge tube and placed in a boiling water bath for 15 min. After cooling in tap water, the mixture was centrifuged at $1,000 \times \mathrm{g}$ for $10 \mathrm{~min}$, and $2 \mathrm{ml}$ of the supernatant was added to $1 \mathrm{ml}$ of $6.7 \mathrm{~g} / \mathrm{l}$ TBA solution in a test tube and placed in a boiling water bath for $15 \mathrm{~min}$. The solution was then cooled with tap water, and its absorbance was measured using a spectrophotometer (UV-1601, Shimadzu, Japan) at 532 NM. The results were expressed as nanomoles per gram protein in the pancreas tissue.
The method for the measurement of SOD was based on the principle in which xanthine reacts with xanthine oxidase to generate superoxide radicals that react with 2-(4-iodophenyl)-3-(4-nitrophenol)-5-phenyltetrazolium chloride to form a red formazan dye. The SOD activity is then measured by the degree of inhibition of this reaction. Superoxide dismutase activity was expressed as units per gram protein [10]. The CAT activity was quantified in pancreas tissue by the method of Aebi. The principle of the method is based on the determination of the rate constant $(s-1, k)$ for $\mathrm{H}_{2} \mathrm{O}_{2}$ decomposition at $240 \mathrm{~nm}$. Results were expressed as $k$ (rate constant) per gram protein [11]. Reduced glutathione (GSH) levels were measured in pancreas, according to the method described by Beutler [12]. The amount of total GSH was determined from a standard curve obtained with known amounts of GSH standards. GSH levels were expressed as units per gram protein.

\section{Assessment of pancreatic mtDNA integrity \\ Isolation of mitochondria}

Mitochondria were extracted by differential centrifugations [13]. One part (100 mg) of tissue was homogenized in $0.25 \mathrm{M}$ sucrose in $0.7 \mathrm{M}$ Tris- $\mathrm{HCl}$ buffer $(\mathrm{pH}=7.4)$ at $1 \mathrm{~g}$ tissue $9 \mathrm{ml}$ of Tris-sucrose. EDTA was added to aid, disruption of cells. Tissue homogenate was spun at $2500 \times \mathrm{g}$ for $10 \mathrm{~min}$ to remove nuclei and unbroken cells. Supernatant fluid was decanted into centrifuge tubes and spun at $10000 \times \mathrm{g}$ for $10 \mathrm{~min}$ to form primary mitochondrial pellets. Supernatant fluid was decanted and the pellet was gently resuspended in $10 \mathrm{ml}$ of Tris-sucrose for washing. The pellet was recentrifuged and supernatant fluid was decanted. This washing cycle was repeated several times to improve the degree of mitochondrial purity. The final mitochondrial pellet was resuspended $(1 \mathrm{ml}$ Tris-sucrose/1 g of original sample).

\section{Isolation of mtDNA}

The mtDNA samples were subjected to $1 \%$ agarose gel electrophoresis at $4 \mathrm{~V} / \mathrm{cm}$ using TAE solution (40 mM Tris-acetate, pH 8.0, 1 mm EDTA) as a running buffer. The gel was stained with $0.5 \mu \mathrm{g} / \mathrm{ml}$ ethidium bromide and photographed by the G:Box Gel Documentation system (Syngene, USA) [14]. Furthermore, mtDNA quantity and purity were determined using NanoDrop 1000 spectrophotometer V3.7 (Thermo Fisher Scientific Inc., Wilmington, DE, USA).

\section{DNA laddering assay}

Endogenous endonucleases, a group of enzymes that cleave double-stranded DNA in the 
linker region between nucleosomes, generating mono- and oligonucleosomes of $180 \mathrm{bp}$ or multiples, are one of the characteristics of apoptosis. To assess endonuclease-dependent ladder-like DNA fragmentation by gel electrophoresis, genomic DNA was extracted from the tissue by Jena Bioscience (Germany) according to the manufacturer's guidelines then loaded onto agarose gel (15 $\mu \mathrm{g} /$ lane). DNA laddering was determined by constant voltage mode electrophoresis (in a large submarine at $4 \mathrm{~V} / \mathrm{cm}$, for $4 \mathrm{~h}$ ) on $1.5 \%$ agarose gel containing $0.5 \mu \mathrm{g} / \mathrm{ml}$ ethidium bromide. A $1 \mathrm{kbp}$ ladder served as a DNA base pair marker [15]. Gels were visualized by the G:Box Gel Documentation system (Syngene, USA).

\section{Histopathological examination}

The injury of the pancreas was evaluated by histological examination of tissue sections $(5 \mathrm{~mm})$ fixed in $4 \%$ formaldehyde, embedded in paraffin, and then cut into 3-5 $\mu \mathrm{m}$ sections and stained with hematoxylin-eosin ( $\mathrm{HE})$ staining. One pathologist experienced in pancreatic pathology performed the histopathological evaluation blindly. Edema, vacuolization, polymorphonuclear neutrophil (PMN) infiltration, hemorrhage and necrosis were assessed in detail, and the changes were represented as follows: $0=$ normal, $1=$ low grade, $2=$ moderate, and $3=$ severe .

The histological grading of edema was made using a scale ranging from 0 to $3(0=$ no edema) $(1=$ interlobular edema) $(2=$ interlobular and moderate intralobular edema) ( 3 = interlobular and severe intralobular edema). The PMN infiltration grading: 0 = absent, 1 = scarce perivascular infiltration, 2 = moderate perivascular and scarce diffuse infiltration, and 3 = abundant diffuse infiltration. Vacuolization was graded depending on the appropriate percentage of acinar cells involved (0 = absent, 1 less than 25\%, $2=25-50 \%$, and 3 more than $50 \%$ ). Hemorrhage grading: $0=$ absent, $1=1-2$ hemorrhagic foci/slide, $2=3-5$ hemorrhagic foci/slide, and 3 = more than 5 hemorrhagic foci/slide. The necrosis grading scale was as follows: $0=$ absent, $1=$ less than $15 \%$ of pancreatic cell involvement, $2=15-35 \%$ of pancreatic cell involvement and $3=$ more than $35 \%$ of pancreatic cell involvement [16].

\section{Immunohistochemical analysis}

The detection of cells that expressed bcl-2, Bax and caspase- 3 proteins relied on immunohistochemistry based on a streptavidin biotin peroxidase method (Biogenex, San Ramon, CA, USA) as described before $[17,18]$. Four-micrometer thick paraffin sections were used, and primary antibodies included the following: monoclonal antibody to bcl-2 (DAKO, CA, USA, at a dilution of $1: 40$ ), polyclonal antibody to Bax (Santa Cruz, USA, at a dilution of $1: 1500$ ), and polyclonal antibody to caspase-3 (Santa Cruz, USA, at a dilution 1 : 150). All incubations were performed for $30 \mathrm{~min}$ at room temperature. Diaminobenzidine (Sigma Fast 3,3'-diaminobenzidine tablets, D-4293; Sigma, St. Louis, MO, USA) was used as the chromogen. Cytoplasmic staining for bcl-2, cytoplasmic and membranous staining for Bax, and cytoplasmic staining for caspase-3 were considered positive.

The integral optical density (IOD) of immunohistochemical intensity was then calculated using Image-Pro Plus 6.0 software. Each value represents IOD counted as a high-power view (400x) by a microscope. The mean value represents the average number derived from the 5 high-power fields of each case [19].

\section{Statistical analysis}

Data were expressed as mean \pm SD and analyzed using the Statistical Package of Social Sciences (SPSS program, version 17, SPSS Inc., Chicago, IL, USA). The difference of mean values among groups was assessed by using one-way analysis of variance (ANOVA) followed by Bonferroni's multiple comparison test. All $p$ values reported are two-tailed, and $p<0.05$ was considered significant. All possible comparisons were made between groups.

\section{Results}

\section{Renal functions (serum creatinine and BUN)}

Table I shows the results of serum creatinine and BUN in the study groups at day 1, day 3 and day 7 after ischemia. Compared to the sham group, serum creatinine and BUN were significantly higher in the I/R group at day 1 , day 3 and day 7 after ischemia $(p<0.05)$, while the treated group was lower than the I/R group at day 1 , day 3 and day 7 after ischemia $(p<0.05)$.

\section{Pancreatic functions (serum amylase,} fasting insulin) and fasting blood glucose

Compared to the sham group, serum amylase showed a significant increase in the I/R group at day 1 and day 3 after ischemia. The treated group showed a significant difference compared to the I/R group at different periods.

Compared to the sham group, fasting insulin showed a significant decrease in the I/R group only at day 1 , while it showed a significant increase at day 3 . The treated group showed a significant difference compared to the I/R group at day 1 and day 3 .

Fasting blood glucose showed a statistically significant difference between sham and I/R groups 
Noha M. Abogresha, Sahar Mansour Greish, Eman Z. Abdelaziz, Waleed F. Khalil

Table I. Renal functions, pancreatic functions and blood glucose

\begin{tabular}{|c|c|c|c|c|c|c|}
\hline Day & Groups & $\begin{array}{c}\text { Creatinine } \\
{[\mathrm{mg} / \mathrm{dl}]}\end{array}$ & $\begin{array}{c}\text { BUN } \\
{[\mathrm{mg} / \mathrm{dl}]}\end{array}$ & $\begin{array}{c}\text { Amylase } \\
{[\mathrm{U} / \mathrm{dl}]}\end{array}$ & $\begin{array}{l}\text { Fasting insulin } \\
\text { [pmol/l] }\end{array}$ & $\begin{array}{l}\text { Fasting blood } \\
\text { glucose }[\mathrm{mg} / \mathrm{dl}]\end{array}$ \\
\hline \multirow[t]{4}{*}{1} & Sham group & $0.63 \pm 0.05$ & $20.71 \pm 0.75$ & $53.16 \pm 5.84$ & $29.51 \pm 0.53$ & $42.33 \pm 2.16$ \\
\hline & I/R group & $0.86 \pm 0.08^{*}$ & $27.52 \pm 2.16^{*}$ & $74.00 \pm 6.72^{*}$ & $20.65 \pm 1.81^{*}$ & $108.50 \pm 5.64^{*}$ \\
\hline & Treated group & $0.71 \pm 0.02^{\dagger}$ & $20.73 \pm 1.17^{\dagger}$ & $50.33 \pm 7.71^{\dagger}$ & $26.81 \pm 4.08^{\dagger}$ & $57.66 \pm 2.25^{\star \dagger}$ \\
\hline & Value of $p$ & 0.012 & 0.013 & 0.012 & 0.014 & 0.015 \\
\hline \multirow[t]{4}{*}{3} & Sham group & $0.81 \pm 0.07$ & $24.83 \pm 0.98$ & $56.33 \pm 4.03$ & $32.66 \pm 1.50$ & $75.83 \pm 2.31$ \\
\hline & I/R group & $1.11 \pm 0.14^{*}$ & $32.50 \pm 1.87^{*}$ & $74.00 \pm 6.00^{*}$ & $48.51 \pm 6.85^{*}$ & $38.38 \pm 3.12^{\star}$ \\
\hline & Treated group & $0.70 \pm 0.07^{\dagger}$ & $20.50 \pm 1.87^{\star \dagger}$ & $62.66 \pm 9.52^{\dagger}$ & $31.33 \pm 6.59^{\dagger}$ & $48.50 \pm 4.23^{\star \dagger}$ \\
\hline & Value of $p$ & 0.013 & 0.012 & 0.012 & 0.015 & 0.016 \\
\hline \multirow[t]{4}{*}{7} & Sham group & $0.75 \pm 0.05$ & $22.66 \pm 1.63$ & $57.00 \pm 5.86$ & $39.95 \pm 0.88$ & $88.66 \pm 20.64$ \\
\hline & I/R group & $1.35 \pm 0.18^{*}$ & $32.33 \pm 4.71^{*}$ & $53.66 \pm 5.83$ & $39.83 \pm 8.91$ & $32.50 \pm 3.78^{\star}$ \\
\hline & Treated group & $0.82 \pm 0.07^{\dagger}$ & $29.83 \pm 2.78^{*}$ & $61.50 \pm 3.39^{\dagger}$ & $40.33 \pm 6.82$ & $93.33 \pm 6.02^{\dagger}$ \\
\hline & Value of $p$ & 0.012 & 0.015 & 0.014 & 0.014 & 0.013 \\
\hline
\end{tabular}

All data are expressed as mean $\pm S D$ and were analyzed using one-way ANOVA and Bonferroni post-hoc test. ${ }^{*}$ Compared to sham group at $p<0.05$. ${ }^{\dagger}$ Compared to I/R group at $p<0.016$. BUN - blood urea nitrogen.

A

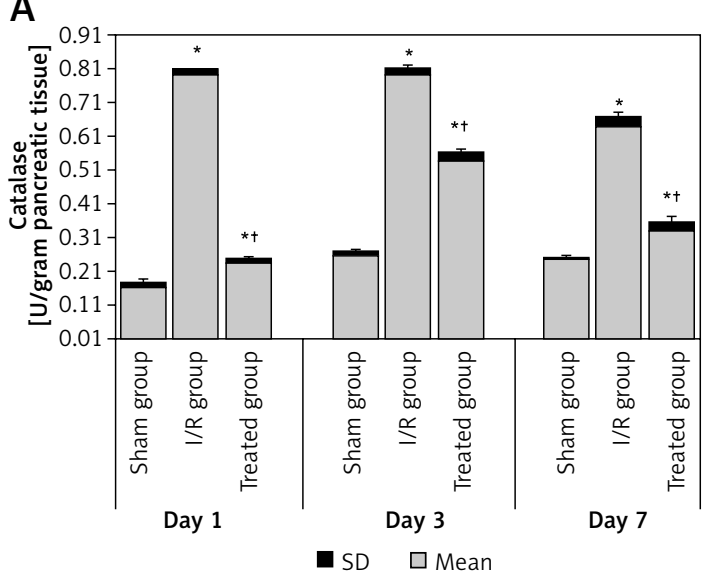

C

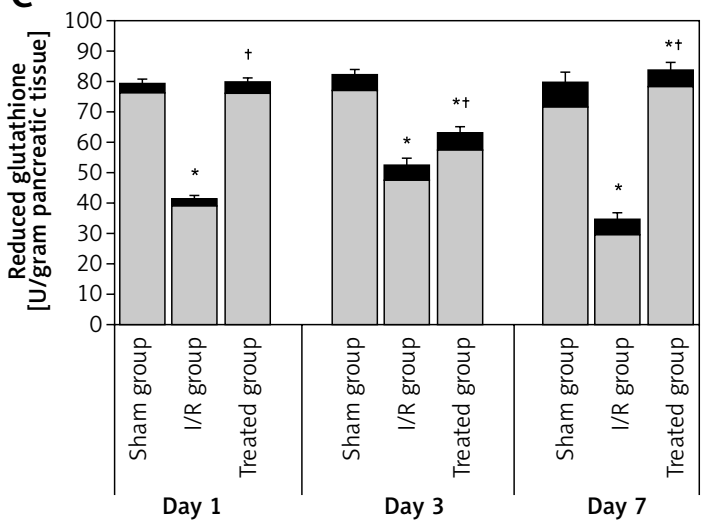

B

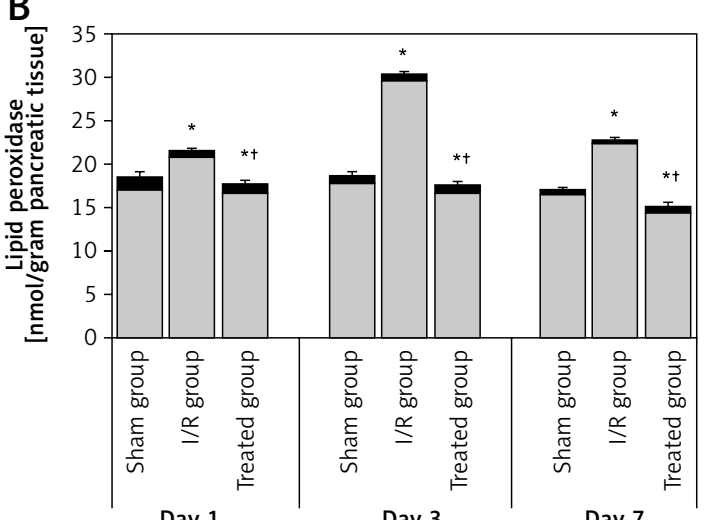

Day 3

Day 7

D

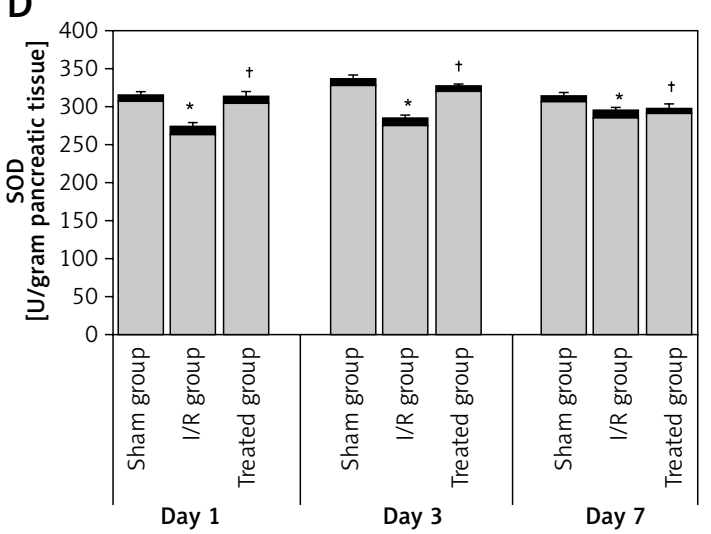

Figure 1. Pancreatic oxidative stress level in the study groups: A - catalase, B - lipid peroxidase, C - reduced glutathione, $\mathbf{D}-\mathrm{SOD}$. All data are expressed as mean \pm SD and were analyzed using one-way ANOVA and Bonferroni post-hoc test

${ }^{*}$ Compared to sham group at $p<0.05 .{ }^{\dagger}$ Compared to I/R group at $p<0.05$. 
at different periods of follow-up. Also, the treated group showed a significant difference compared to the I/R group at different periods (Table I).

\section{Oxidative stress markers in pancreatic tissues}

The results of the catalase, lipid peroxidase, reduced glutathione and SOD analyses are shown in Figure 1. Compared to the sham group, the levels of catalase and lipid peroxidase were significantly higher in the pancreatic tissue of the I/R group at day 1 , day 3 and day 7 after ischemia. The treated group showed a significant decrease compared to the I/R group at different periods (Figures $1 \mathrm{~A}-\mathrm{B}$ ).

Reduced glutathione and SOD concentration in pancreatic tissues were significantly lower in the I/R group when compared to the sham group at day 1 , day 3 and day 7 after ischemia. Also, the treated group showed a significant increase compared to the I/R group at different periods (Figures $1 \mathrm{C}-\mathrm{D}$ ).

\section{Histopathological examination and immunostaining}

Table II shows the results of pancreatic weight and damage score. Compared to the sham group, the weight of the pancreas was significantly increased in the I/R group at day 1, day 3 and day 7 after ischemia. However, the treated group showed a significant decrease in pancreatic weight only at day 7 after ischemia.

Regarding the histological damage score, pancreatic edema, leucocytic infiltration, and vacuolization were significantly higher in the I/R group at different periods of follow-up when compared to the sham group. However, the group treated with vitamin $C$ showed significant improvement regarding edema, leucocytic infiltration, and vacuolization at different periods. All groups showed no pancreatic hemorrhage or necrosis (Figure 2).
The expression levels of the apoptosis-related genes, including Bax (apoptotic gene), Bcl-2 (anti-apoptotic gene) and caspase-3, which is an apoptosis coordination enzyme, were analyzed by immunohistochemistry for the pancreatic tissues in the experimental groups at day 7 . The expression of $\mathrm{BCl}-2$ in the I/R group was significantly decreased, while the expression of Bax and caspase- 3 in the I/R group was significantly increased when compared to the sham and treated groups. This means that the apoptosis increased in the I/R group and was improved by vitamin $C$ treatment (Figure 3).

\section{Mitochondrial DNA integrity and fragmentation of nuclear DNA}

In the sham group, the intact form of mtDNA was electrophoresed as a major band of approximately $16.5 \mathrm{~kb}$ (lane 1). On the other hand, ischemia/reperfusion injury markedly reduced the amounts of intact mtDNA in the pancreatic tissue (lane 2). However, mtDNA from rats treated with vitamin $C$ at day 1 , day 3 and day 7 (lanes 3 , $4,5)$ was electrophoresed in its intact form (Figure 4 A).

The ischemia/reperfusion resulted in a marked decrease in the amount of intact nDNA in pancreatic tissues (Figure $4 \mathrm{~B}$ ). Moreover, dramatic oligonucleosome-length degradation of DNA was observed, characterized by mixed smearing and laddering (lane 5). No appreciable fragmentation of nDNA was found to occur in animals treated with vitamin $C$ at day 1 , day 3 and day 7 (lanes $1,2,3)$. Also, the sham group did not show any fragmentation of nDNA (lane 4).

\section{Discussion}

Remote effects of renal IR injury of the pancreas still have unclear aspects. Hence, this study investigated the effects of renal IR on the pancre-

Table II. Pancreatic weight and histopathological damage score

\begin{tabular}{|lccccccc|}
\hline Day & Groups & Weight & Edema & $\begin{array}{c}\text { Leucocytic } \\
\text { infiltration }\end{array}$ & Vacuolization & Hemorrhage & Necrosis \\
\hline 1 & Sham group & $0.65 \pm 0.05$ & $0-1$ & 0 & 0 & 0 & 0 \\
\cline { 2 - 8 } & I/R group & $0.88 \pm 0.19^{*}$ & $1-2^{*}$ & $0-1^{*}$ & $1^{*}$ & 0 & 0 \\
\cline { 2 - 8 } & Treated group & $0.80 \pm 0.00$ & 2 & $1 \dagger$ & 1 & 0 & 0 \\
\hline 3 & Sham group & $0.60 \pm 0.00$ & 0 & $0-1$ & 0 & 0 & 0 \\
\cline { 2 - 8 } & I/R group & $0.86 \pm 0.20^{*}$ & $3^{*}$ & $2-3^{*}$ & $2^{*}$ & 0 & 0 \\
\hline 7 & Treated group & $0.80 \pm 0.14$ & $1-2^{\dagger}$ & $2^{\dagger}$ & $1^{+}$ & 0 & 0 \\
\cline { 2 - 8 } & Sham group & $0.60 \pm 0.00$ & 0 & $0-1$ & 0 & 0 & 0 \\
\cline { 2 - 8 } & I/R group & $0.80 \pm 0.14^{*}$ & $2^{*}$ & $1^{*}$ & $1^{*}$ & 0 & 0 \\
\hline & Treated group & $0.51 \pm 0.11^{\dagger}$ & $1^{\dagger}$ & $0^{+}$ & $0-1$ & 0 & 0 \\
\hline
\end{tabular}

Data of pancreatic weight are expressed as mean $\pm S D$ and were analyzed using one-way ANOVA and Bonferroni post-hoc test. ${ }^{*}$ Compared to sham group at $p<0.016$. ${ }^{\dagger}$ Compared to I/R group at $p<0.016$. Numbers represent the predominant grading of pancreatic damage. 
Day 1
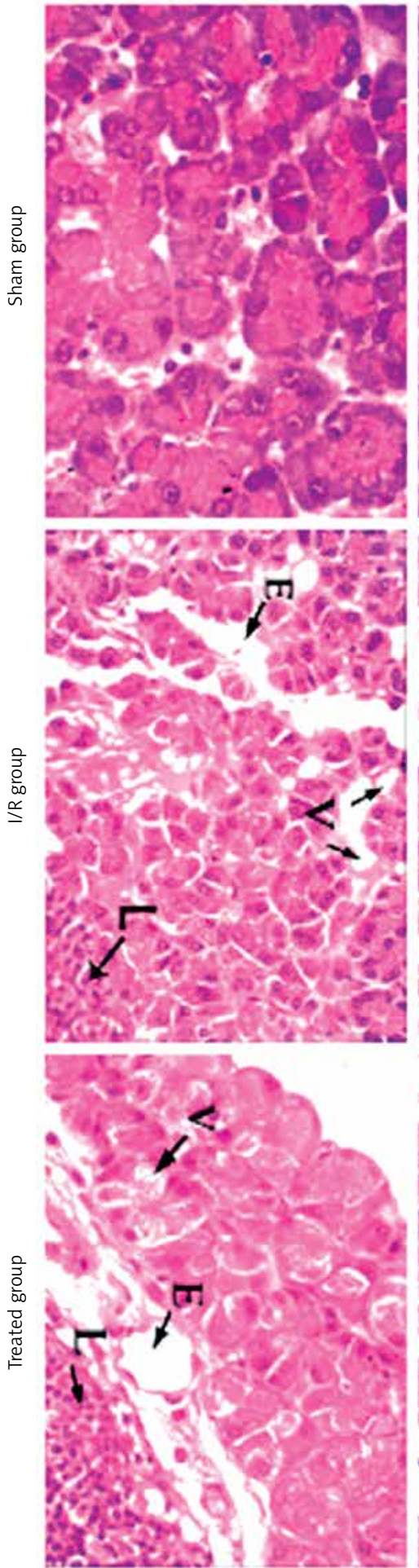

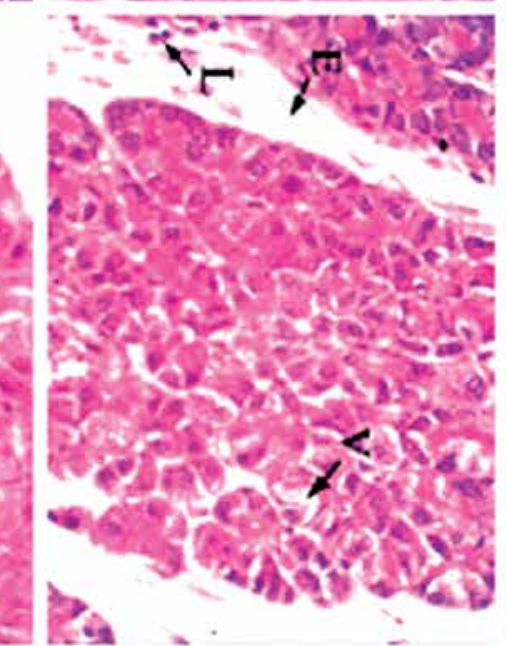

Day 3
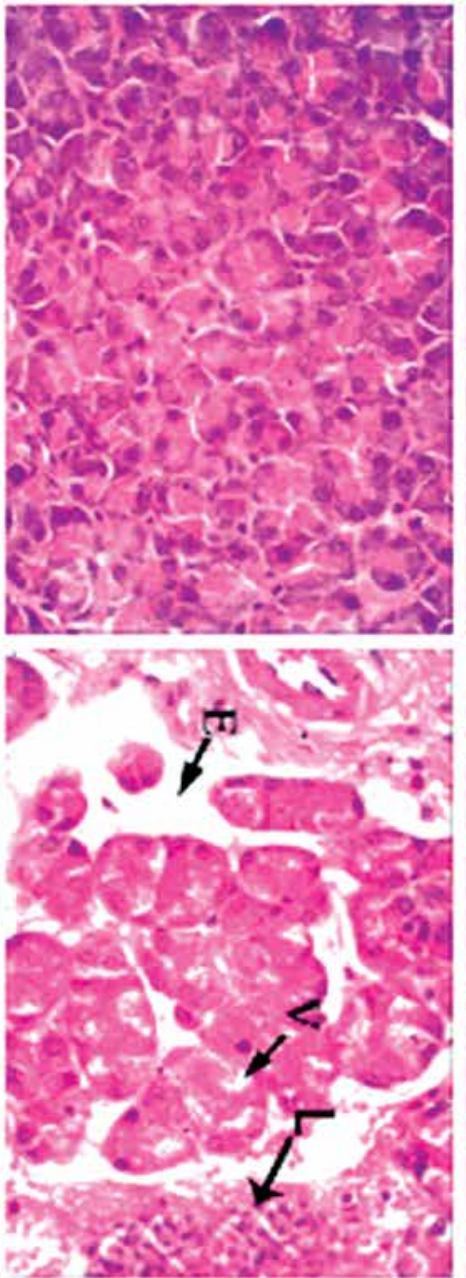

Day 7
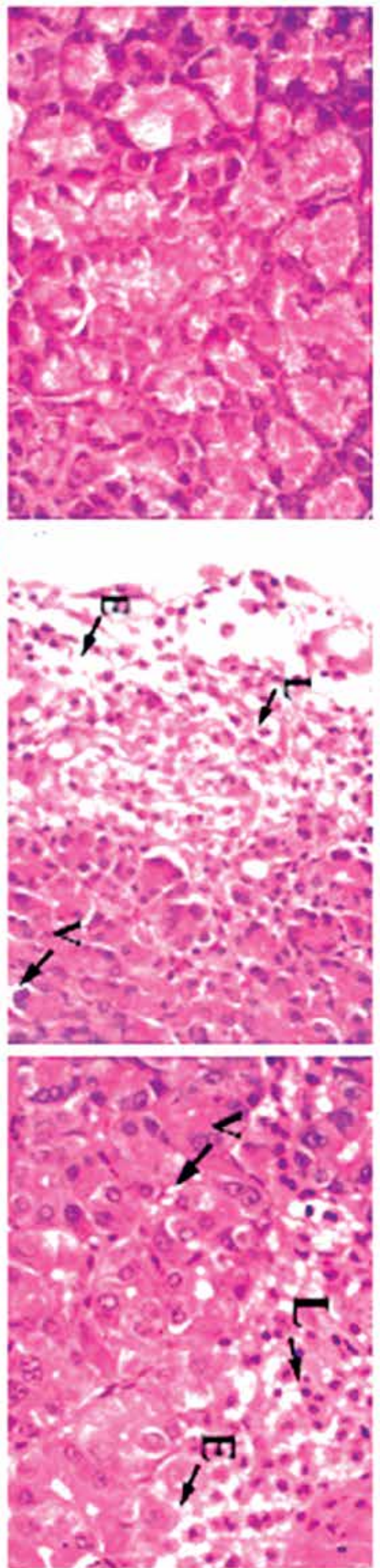

Figure 2. Microphotographs in pancreatic tissue of sham, ischemic and treated groups at day 1 , day 3 and day 7 of the experiment $(H+E, 400 \times)$. Sham group shows normal pancreatic tissue. I/R group shows moderate to severe pathological changes. Treated group shows mild pathological changes

$E$-edema, $L$ - leucocytic infiltration, $V$-vacuolization.

as and the effects of vitamin C application after ischemia by assessing some biochemical and histological parameters in a rat model of ischemia reperfusion injury.
Biochemically, compared to the sham group, the serum concentrations of amylase, creatinine and BUN elevated significantly in the I/R group at day 1 and day 3 after ischemia with impairment of 
A

Sham group

足

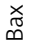

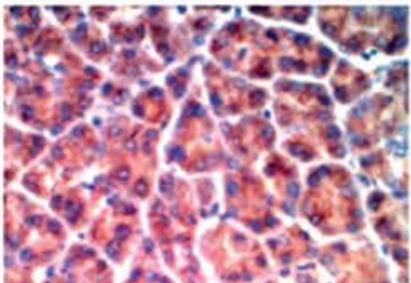

4.

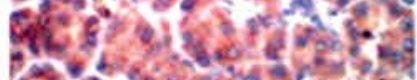

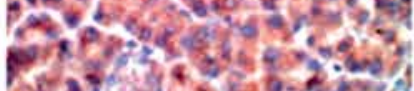

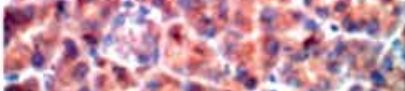

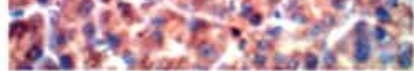

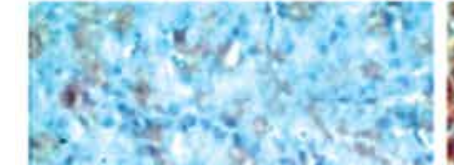

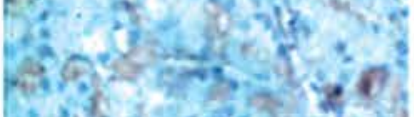

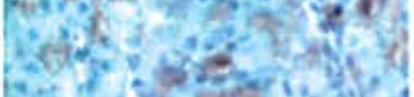

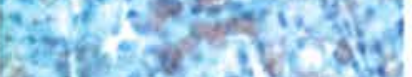

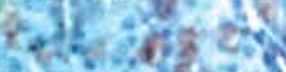

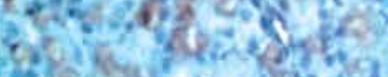

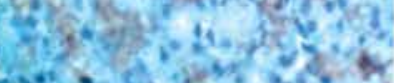

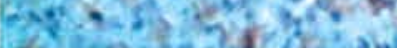

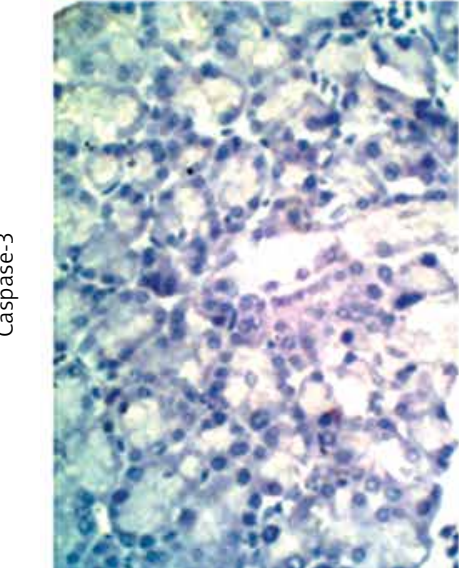

I/R group
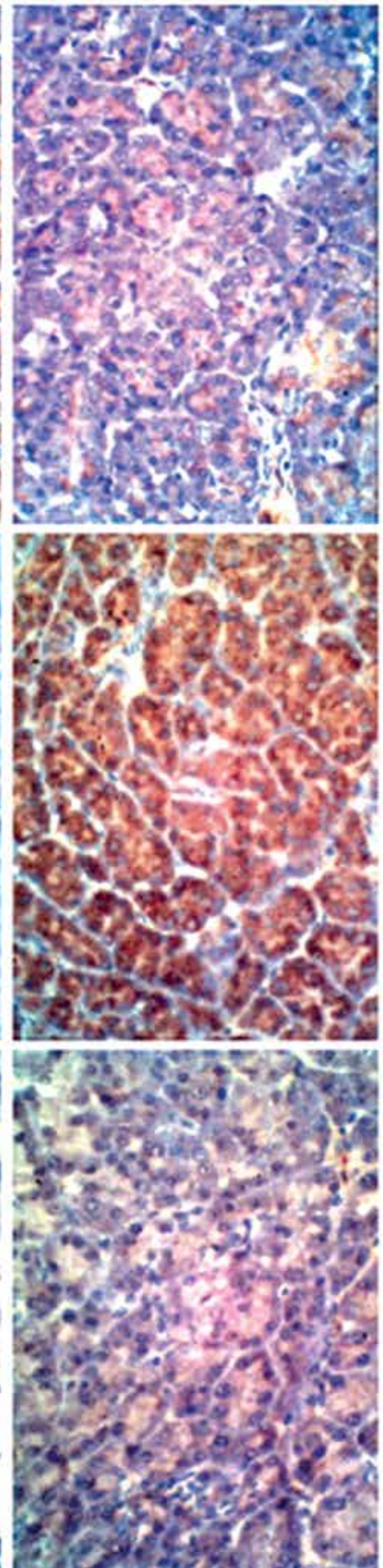

Treated group
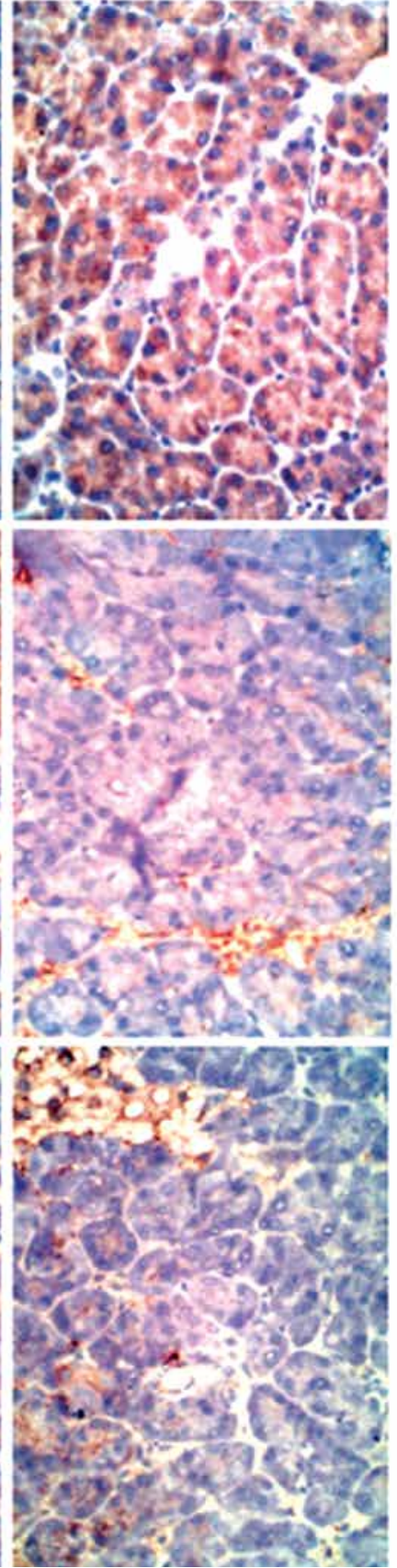

B

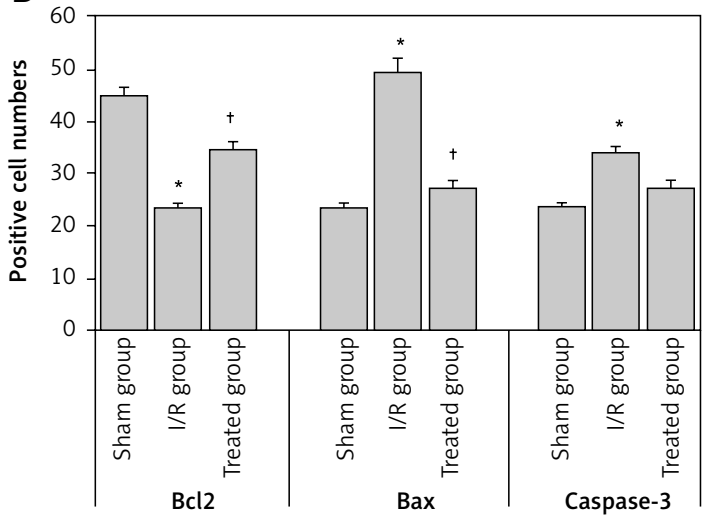

Figure 3. A - Microphotographs showing immunostaining for $\mathrm{BCl}-2, \mathrm{Bax}$ and caspase- 3 in the sham, I/R and treated groups at day 7 of the experiment (IHC, 400x). B - IOD of Bcl-2, Bax and caspase-3 immunohistochemical intensity was quantified by Image-Pro Plus 0.6 software 
A

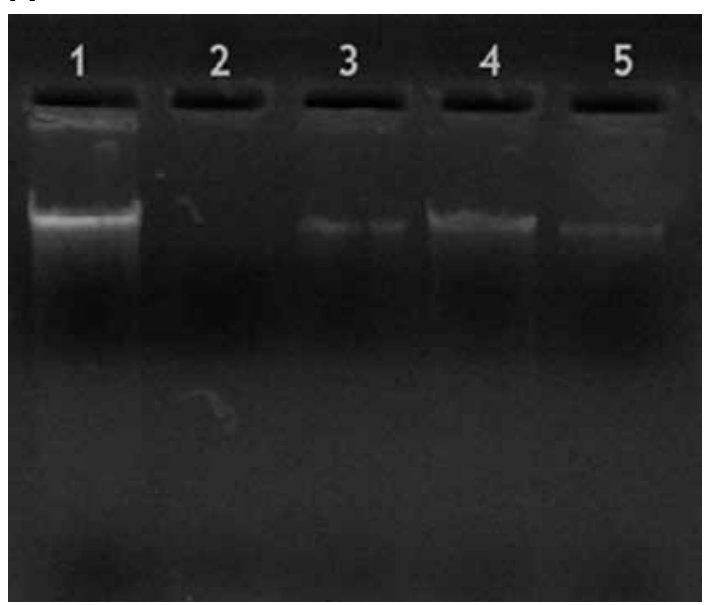

B

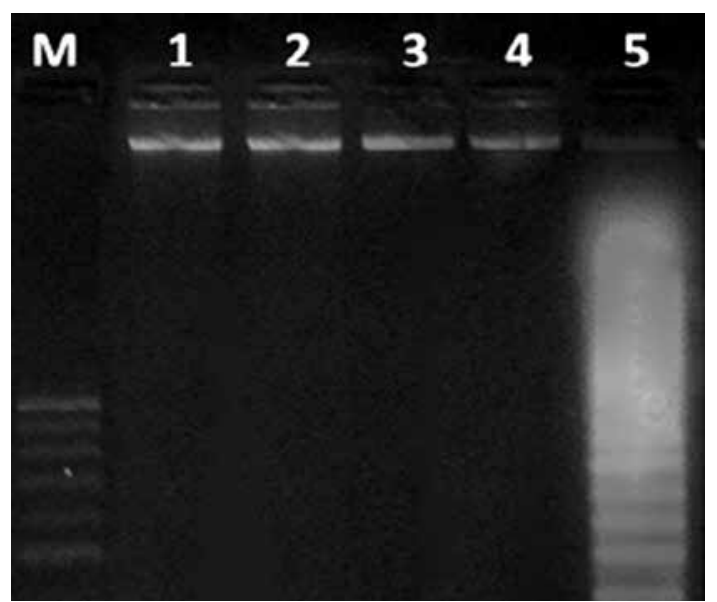

Figure 4. Mitochondrial DNA (mtDNA) and nuclear DNA (nDNA) in the experimental groups. A - mtDNA of the experimental groups, lane 1: intact mtDNA isolated from sham group; lane 2: mtDNA samples isolated from I/R group; lanes 3, 4, 5: mtDNA samples isolated from treated groups; B - integrity of nDNA of the experimental groups. Lane M, 1 kb DNA ladder; lane 4, intact nDNA isolated from sham group; lane 1, lane 2 and lane 3, nDNA sample isolated from treated groups; lane 5, nDNA isolated from I/R group

other pancreatic function (fasting insulin showed a significant decrease in the ischemic group only at day 1 , while it showed a significant increase at day 3$)$. These results were congruent with other studies $[3,20]$. In the treated group, vitamin C suppressed the rise in serum amylase, creatinine and BUN, and improved other pancreatic functions compared to the sham group at different periods. Moreover, fasting blood glucose showed a statistically significant difference between sham and I/R groups at different periods of follow-up. These findings suggest that the pancreatic injury induced by renal ischemia was sufficient to affect the endocrine functions of the pancreas and affect glucose homeostasis. These findings contrast with those of other research, where blood glucose and fasting insulin showed no significant change apart from a significant increase in insulin in the sham group at 1 day after ischemia [3, 20].

All the results of biochemical parameters may be due to the effect of short-term ischemia and the reperfusion method, which generates active oxygen species that injure the endothelium, cause hyperamylasemia and impair the kidney functions. Meanwhile, the inhibition of the rise in serum amylase concentration and restoration of kidney function by treatment with a scavenger of active oxygen, vitamin $\mathrm{C}$, confirms that active oxygen species are involved in the pathogenesis of acute pancreatitis. Regarding the effect of IR injury on the level of lipid peroxidation in pancreatic tissue, in comparison to the sham group, the levels of catalase and lipid peroxidase were significantly higher in the I/R group at different periods after ischemia, with significantly lower levels of reduced glutathione and SOD concentration in the I/R group when compared to the sham group at day 1 , day 3 and day 7 after ischemia. Also, the treated group showed a significant decrease in lipid peroxidative enzymes (Figures $1 \mathrm{~A}-\mathrm{B}$ ) and a significant increase in antioxidant enzymes (Figures $1 \mathrm{C}-\mathrm{D})$ compared to the I/R group in different periods. These results are in accordance with previous studies [21, 22]. Abdollahzad et al. found that every other day supplementation with $250 \mathrm{mg}$ of vitamin C for 12 weeks increased serum vitamin C and reduced MDA levels [22].

On the other hand, other studies have suggested that the increased lipid peroxidation may be due to ischemia that results not only in catabolization of ATP, but also in the conversion of NAD-reduced xanthine hydrogenase into the oxygen radical producing xanthine oxidase $[4,21]$. Reperfusion and reoxygenation of tissue in the presence of hypoxanthine and xanthine oxidase lead to production of aggressive oxygen radicals [4, 21]. Also, Muñoz-Casares et al. stated that IR induced a marked enhancement of oxidative stress [23]. But with the use of vitamin C, the opposite occurs, as it is one of the most important antioxidants that inhibit lipid peroxidation and improve endothelial function [22].

Vitamin C concentration in plasma of humans is tightly controlled, and excess vitamin $\mathrm{C}$ is excreted as a function of dose, being completely saturated at doses of $400 \mathrm{mg}$ daily and higher, producing a steady-state plasma concentration of approximately $80 \mu \mathrm{M}$. Ramón Rodrigo et al. found that intra-arterial administration of high doses of ascorbate abolished the in vivo effects of superoxide anion in the impairment of vascular endothelial function in subjects with essential hypertension. However, in our experimental study, vitamin C in a dosage of $100 \mathrm{mg} / \mathrm{kg}$ dissolved in drinking water 
was able to prevent pancreatic injury and apoptosis after IR injury in rats. The protective effect of low doses of vitamin $C$ in vivo may be referred to its biologic properties, other than that of scavenging ROS, which may have some beneficial effect. The major source of ROS is their enzymatic production via NA$\mathrm{DPH}$ oxidase, an enzyme subjected to down-regulation by vitamin C. In addition, vitamin C prevents the oxidation of tetrahydrobiopterin, a cofactor of (nitric oxide) NO synthase that is highly sensitive to oxidation. When tetrahydrobiopterin is oxidized, endothelial nitric oxide synthase (eNOS) activity becomes uncoupled, resulting in the production of superoxide instead of NO, thus enhancing the oxidative damage [24].

Histologically, pancreatic injury by renal IR is characterized by pancreatic edema, leucocytic infiltration and vacuolization, with no pancreatic hemorrhage or necrosis (Figure 2). These findings are significantly higher in the I/R group at different periods of follow-up when compared to the sham group. However, the group treated with vitamin C showed significant histological improvement at different periods. These results are consistent with the findings of many previous studies [21, 23, 25-27] where the histological analysis showed that IR induced acute pancreatitis with the accumulation of inflammatory infiltrate, disruption of tissue structure, cell necrosis and hemorrhage. These conclude that the pancreas is highly susceptible to oxidative stress induced by IR injury, leading to the development of acute pancreatitis [21, 23], and this damage can be prevented by antioxidants [21].

Regarding mitochondrial DNA (mtDNA) integrity and fragmentation of nuclear DNA (nDNA), $I / R$ injury markedly reduces the amounts of intact mtDNA and nDNA in the pancreatic tissue (lane 2 and Figure $4 \mathrm{~B}$ respectively). Moreover, dramatic oligonucleosome-length degradation of DNA is observed, characterized by mixed smearing and laddering (lane 5). On the other hand, mtDNA from rats treated with vitamin $C$ at different periods after ischemia (lanes 3, 4, 5) was electrophoresed in its intact form (Figure $4 \mathrm{~A}$ ) with no appreciable fragmentation of nDNA (lanes 1, 2, 3). Also, the sham group did not show any fragmentation of nDNA (lane 4). These results are in accordance with Fujimoto et al., who detected DNA breaks of acinar cells by gel electrophoresis, and the numbers strikingly increased at $48 \mathrm{~h}$ after reperfusion [25]. Another study showed that $\beta$-cells are particularly sensitive to ROS because they are low in free-radical quenching enzymes [4]. Therefore, the ability of oxidative stress to damage mitochondria and markedly blunt insulin secretion is not surprising in our study. In the I/R group, the expression of $\mathrm{BCl}-2$ significantly decreased, while the expression of Bax and caspase- 3 significantly increased when compared to the sham and treat- ed groups. This means that apoptosis increased in the ischemic group and was improved by vitamin C treatment. These results are similar to those of $\mathrm{Fu}$ jimoto et al., in which Bax protein, an effector of apoptotic cell death, was expressed in acinar cells after I/R injury of the pancreas in rats [25].

Apoptosis in acinar cells may be one of the specific features of $I / R$ reperfusion. Oxidative stress triggers caspase- 3 activation leading to cell apoptosis. In addition, the balance of anti- and proapoptotic proteins ( $\mathrm{Bcl}-2$ : Bax) in the mitochondria responds dramatically to ROS [26, 27]. Bcl-2 is a kind of inhibitor of cell apoptosis. It can prevent apoptosis induced by free radicals and lipid peroxidation. Bcl-2 has antioxidative characteristics in cells through participating in the reduction process and inhibiting the formation of active oxygen. Bcl-2 also preserved mitochondrial oxidative function [28].

In conclusion, our histological parameters support the biochemical parameters; our results indicate that the massive production of ROS during I/R leads to tissue injury, causing acute pancreatitis and apoptosis. However, these changes can be reduced by concomitant supply of vitamin C as one of the most important antioxidants.

\section{Conflict of interest}

The authors declare no conflict of interest.

\section{References}

1. Laura E, Heitham T. Inflammatory mechanisms of organ crosstalk during ischemic acute kidney injury. Intern J Nephrol 2012; 2012: 505197.

2. Bulent G. Ischemia reperfusion injury in kidney transplantation. Available at: http://www.intechopen.com/ books/kidney-transplantation-new-perspectives/ischemia-reperfusion-injury-in-kidney-transplantation, 2011.

3. Hussein A, Abd-Elkhabir A, Abozahra A, et al. Pancreatic injury secondary to renal ischemia/reperfusion (I/R) injury: possible role of oxidative stress. Physiol Res 2013; 63: 47-55.

4. Marian V, Dieter L, Jan M, Mark T, Milan M, Joshua T. Free radicals and antioxidants in normal physiological functions and human disease. Intern J Biochem Cell Biol 2007; 39: 44-84.

5. Hengartner M, Bryant J. Apoptotic cell death: from worms to wombats ... but what about the weeds? Symp Soc Exp Biol 2000; 52: 1-12.

6. Yousef D, Daryoush M, Alireza G, Ghafour M, Mehrdad N. Protective effect of metformin on cardiomyocytes ischemia-reperfusion (IR) induced apoptosis in rats. J Animal Vet Adv 2012; 11: 108-12.

7. Philchenkov A, Zavelevich M, Kroczak T, Los M. Caspases and cancer: mechanisms of inactivation and new treatment modalities. Exp Oncol 2004; 26: 82-97.

8. Chambial S, Dwivedi S, Shukla KK, John PJ, Sharma P. Vitamin $C$ in disease prevention and cure: an overview. Indian J Clin Biochem 2013; 28: 314-28.

9. Draper HH, Hadley M. Malondialdehyde determination as index of lipid peroxidation. Methods Enzymol 1990; 186: 421-31. 
10. Woolliams JA, Wiener G, Anderson PH, et al. Variation in the activities of glutathione peroxidase and superoxide dismutase and in the concentration of copper in the blood in various breed crosses of sheep. Res Vet Sci 1983; 34: 253-6.

11. Aebi H. Catalase in vitro. Methods Enzymol 1984; 105: 121-6.

12. Beutler E, Duron O, Kelly BM. Improved method for the determination of blood glutathione. J Lab Clin Med 1963; 61: 882-8.

13. Chappel JB, Hansford RG. Subcellular components. Butterworths, London 1969.

14. Chang BJ, Nishikawa M, Sato E, Utsumi K, Inoue M L-Carnitine inhibits cisplatin-induced injury of the kidney and small intestine. Arch Biochem Biophys 2002. 405: 55-64.

15. Sambrook J, Maniatis T, Fritsch F. Molecular cloning, a laboratory manual. Cold Spring Harbor Laboratory Press, Cold Spring Harbor, New York 2001.

16. Warzecha Z, Dembinski A, Ceranowicz P, et al. Immunohistochemical expression of FGF-2, PDGF-A, VEGF and TGFbeta RII in the pancreas in the course of ischemia/ reperfusion- induced acute pancreatitis. J Physiol Pharm 2004; 55: 791-810.

17. Salakou S, Tsamandas AC, Bonikos D, Papapetropoulos T, Dougenis D. The potential role of bcl-2, bax and Ki67 expression in thymus of patients with myasthenia gravis, and their correlation with clinicopathologic parameters. Eur J Cardiothoracic Sur 2001; 20: 712-72.

18. Scopa CD, Vagianos C, Kardamakis D, Kourelis T, Kalofonos $\mathrm{H}$, Tsamandas AC. bcl-2/bax ratio as a predictive marker for therapeutic response to radiotherapy in patients with rectal cancer. App Immunohistochem Mol Morph 2001; 9: 329-34

19. Salakou S, Kardamakis D, Tsamandas AC, et al. Increased $\mathrm{Bax} / \mathrm{Bcl}-2$ ratio up-regulates caspase- 3 and increases apoptosis in the thymus of patients with myasthenia gravis. In Vivo 2007; 21: 123-32.

20. Tamura K, Manabe T, Kyogoku T, Andoh K, Ohshio G, Tobe T. Effect of postischemic reperfusion on the pancreas. Hepatogastroenterology 1993; 40: 452-6.

21. Yildirim A, Tuncer MC, Pamukçu O, Aktas A, Akkus M. Effects of ischemia/reperfusion on beta cells of pancreas and protective effects of melatonin treatment. Int J Morphol 2009; 27: 519-25.

22. Abdollahzad H, Eghtesadi S, Nourmohammadi I, KhademAnsari M, Nejad-Gashti H, Esmaillzadeh A. Effect of vitamin C supplementation on oxidative stress and lipid profiles in hemodialysis patients. Int I Vitam Nutr Res 2006; 79: 281-7.

23. Muñoz-Casares F, Padillo F, Briceño J, et al. Melatonin reduces apoptosis and necrosis induced by ischemia/ reperfusion injury of the pancreas. J Pineal Res 2006; 40: 195-203.

24. Rodrigo R, Hasson D, Prieto J, et al. The effectiveness of antioxidant vitamins $C$ and $E$ in reducing myocardial infarct size in patients subjected to percutaneous coronary angioplast y (PREVEC Trial): study protocol for a pilot randomized double-blind controlled trial. Trials 2014; 15: 192

25. Fujimoto K, Hosotani R, Wada $M$, et al. Ischemia-reperfusion injury on the pancreas in rats: identification of acinar cell apoptosis. J Surg Res 1997; 71: 127-36.

26. Obermaier R, Benz S, Kortmann B, Benthues A, Ansorge $N$, Hopt UT. Ischemia/reperfusion induced pancreatitis in rats: a new model of complete normothermic in situ ischemia of a pancreatic tail-segment. Clin Exp Med 2001; 1: 51-9.
27. CemKoçkar M, Rana S, Efkanz U, et al. Effects of erdosteine, vitamin $C$ and $E$ on ischemia/reperfusion induced pancreatic injury in rats. Nobel Med 2012; 8: 49-54.

28. Chen CC, Liu ZM, Wang HH, He W, Wang Y, Wu WD. Effects of ulinastatin on renal ischemia-reperfusion injury in rats. Acta Pharmacol Sin 2004; 25: 1334-40. 